\title{
Different requirement of immunity pathway components by oomycete effectors-induced cell death
}

\author{
Xiaohua Dong, Gan Ai, Chuyan Xia, Weiye Pan, Zhiyuan Yin and Daolong Dou* (10
}

\begin{abstract}
Plant pathogenic oomycete species pose a worldwide threat to crop production and ecosystems. During infection, oomycete pathogens secrete a series of effectors to manipulate plant immunity. Many of these effectors, which are indicated as avirluence gene candidates, will use components of immunity pathway to induce cell death in plants. This response given by plants is known as effector-triggered immunity (ETI). The identification of avirulence genes from pathogenic oomycete species opens a way to investigating their virulence function and uncovering related $R$ gene repertoires in resistant plants. In this study, we screened eight cell death-inducing effectors from oomycete species in N. benthamiana and tested the requirements of ETI signaling components to induce cell death. SGT1 was required for PsAvh163- and PCRXLR25-mediated cell death, while silencing NbHSP90 abolished PcRXLR25-, PsAvh163-, PsAvh241- and PsCRN63-triggered cell death. The cell death induced by the tested effectors does not depend on EDS1, NDR1, NRG1 and ADR1. PCRXLR25- and PsAvh163-induced cell death was found to require NRC2/3/4, indicating that these two effectors are avirulence protein candidates. Finally, we found that auto-activated NRC2/3/4 also required SGT1 and HSP90 to induce hypersensitive response.
\end{abstract}

Keywords: Phytophthora, Effector-triggered immunity, RXLR, CRN, R gene

\section{Background}

Oomycete pathogens continue to hamper crop production and damage ecosystems on a global scale (Pais et al. 2013). A notorious group of pathogens are found within the Phytophthora genus and Pythium genus, among which members such as Phytophthora infestans and Phytophthora sojae cause severe yield losses in potato, tomato and soybean crops, while others, such as Pythium ultimum is a rapidly emerging pathogen with a broad host range (Kamoun et al. 2015). Oomycetes belong to heterokont/chromist clade (Riisberg et al. 2009), within the 'Straminipila-Alveolata-Rhizaria' superkingdom (Burki et al. 2008). The common management method

*Correspondence: ddou@njau.edu.cn

College of Plant Protection, Academy for Advanced Interdisciplinary

Studies, Nanjing Agricultural University, Nanjing 210095, China for controlling pathogenic fungi may fail to prevent infections of oomycete pathogens due to the great diversity between these two types of pathogens. Therefore, there is an urgent need to understand the mechanisms underpinning the parasitism of this important group of eukaryotes.

For successful colonization in host plants, oomycete pathogens deliver diverse groups of effectors into plant cells to subvert host immunity (Dou and Zhou 2012). Oomycete pathogens mostly secrete two classes of effectors: apoplastic effectors such as necrosis and ethyleneinducing peptide-like proteins (NLPs), and cytoplasmic effectors such as RXLRs (Arg-X-Leu-Arg, where X is any amino acid) and CRNs (crinkling and necrosis proteins) (Dou and Zhou 2012). Specifically, hundreds of effectors are encoded by oomycete pathogens and many of them trigger hypersensitive response (HR)-like phenotype in original author(s) and the source, provide a link to the Creative Commons licence, and indicate if changes were made. The images or other third party material in this article are included in the article's Creative Commons licence, unless indicated otherwise in a credit line to the material. If material is not included in the article's Creative Commons licence and your intended use is not permitted by statutory regulation or exceeds the permitted use, you will need to obtain permission directly from the copyright holder. To view a copy of this licence, visit http://creativecommons.org/licenses/by/4.0/. 
plants (Dou and Zhou 2012). Notably, many RXLR effectors are avirluence (AVR) proteins (Rehmany et al. 2005). For example, AVR1, AVR3a, AVRblb1 and AVRvnt1 are RXLR effectors identified in P. infestans (Ballvora et al. 2002; van der Vossen et al. 2003; Huang et al. 2005; Bos et al. 2006; Foster et al. 2009).

Plants use their sophisticated immune system to antagonize the invasion of external pathogens. Plants employ two main regulatory strategies to defend themselves against pathogens: pattern-triggered immunity (PTI) and effector-triggered immunity (ETI) (Jones and Dangl 2006). PTI requires membrane-localized pattern recognition receptors to recognize pathogen- or microbe-associated molecular patterns (Zipfel 2008). However, virulent pathogens can secrete effectors to escape from the recognition by pattern-recognition receptors and therefore reach the aim of manipulating host immunity (Tsuda and Katagiri 2010). To address this problem, ETI, which is mediated by disease resistance $(\mathrm{R})$ proteins, can directly or indirectly recognize the presence of effectors secreted by pathogens (Tsuda and Katagiri 2010). Compared with PTI, ETI induces a stronger and faster defense response against pathogens and is often accompanied by local cell death, a characteristic feature of the hypersensitive response (HR) (Dodds and Rathjen 2010).

Upon pathogen recognition, conformational changes and/or translocation of the sensors would activate the downstream immunity signaling pathways. Thence, misfolded or used sensors become a threat to host cells and must be inactivated and discarded immediately to avoid inappropriate activation of downstream pathways. Fortunately, several critical components of the ETI pathway have been identified to correct these misfolded and overused sensors, such as SGT1 and HSP90 (Shirasu 2009). SGT1-HSP90 pair, a structurally and functionally conserved chaperone complex in eukaryotes, is required for the maintenance of nucleotide-binding leucine-rich repeat (NLR)-type sensors in a correct state (Shirasu 2009). Another ETI regulator, ENHANCED DISEASE SUSCEPTIBILITY 1 (EDS1), is a conserved lipase-like protein that can transduce signals from pathogen-activated intracellular NLR receptors to transcriptional defense response and host cell death (Dongus and Parker 2021). In addition, NONRACE-SPECIFIC DISEASE RESISTANCE (NDR1), a positive plant immunity regulator, is required for both PTI and ETI (Knepper et al. 2011b; McNeece et al. 2017). NDR1 is also important for mediating electrolyte leakage because of its plasma membrane-localization (Knepper et al. 2011a).

NLR-mediated immune responses often require the presence and activity of so called 'helper' NLRs (hNLRs) (Wu et al. 2017; Qi et al. 2018). There are three described hNLR families, all encoding coiledcoil (CC)-NLRs (CNLs): the ACTIVATED DISEASE RESISTANCE 1 (ADR1) family (Bonardi et al. 2011), the N REQUIRED GENE 1 (NRG1) family (Peart et al. 2005) and HR-associated cell death (NRC) family (NBLRR protein) (Gabriels et al. 2007). It seems that hNLRs serve as downstream signaling hubs for a diverse array of senser NLRs (Jubic et al. 2019).

In this study, we tested the requirement of ETI components for eight oomycete effectors to induce cell death in $N$. benthamiana. ETI-related genes were silenced by virus-induced gene silencing (VIGS) and effectors were then expressed in silenced leaves. SGT1 was found to be essential for INF1, VdEIX3, PcRXLR25 and PsAvh163 to induce cell death in $N$. benthamiana, while silencing NbHSP90 abolished PcRXLR25-, PsAvh163-, PsAvh241- and PsCRN63-triggered cell death. HR induced by PcRXLR25 and PsAvh163 required NRC2/3/4. No effectors induced cell death through EDS1, NDR1, NRG1 and ADR1. PcRXLR25 and PsAvh163 depended on both SGT1/HSP90 and $\mathrm{NRC} 2 / 3 / 4$ to induce $\mathrm{HR}$, and this inspired us to investigate the relationship of $\mathrm{NRC} 2 / 3 / 4$ with $\mathrm{SGT} 1$ and HSP90. Moreover, we found auto-activated NRC2/3/4 required SGT1 and HSP90 to induce HR. Interestingly, however, there was no interaction between NRC2/3/4 and SGT1 or HSP90 by conducting a luciferase complementation assay.

\section{Results}

Eight oomycete effectors induce HR-like phenotype in $N$. benthamiana

Our laboratory has reported that many effectors from oomycete species induce cell death in $N$. benthamiana (Li et al. 2019; Ai et al. 2020). To figure out whether the oomycete effector-induced cell death requires ETI components, a transient expression screening was conducted. Eight effectors listed as INF1, PoNLP5, PoNLP7, PcRxLR25, PsAvh105, PsAvh163, PsAvh241 and PsCRN63 were chosen in this assay (Additional file 1: Table S1). VdEIX3, a fungal effector that induces pattern-triggered immunity (PTI), was selected as a control (Yin et al. 2021).

To confirm that these effectors are able to induce cell death, we transiently expressed them in leaves of $N$. benthamiana. GFP and VdEIX3 were used as negative and positive control, respectively. All the eight effectors and VdEIX3 induced obvious cell death in infiltration sites (Fig. 1a), while leaves expressing GFP did not. Confocal images and Western blot assay indicated that all the proteins were expressed correctly (Fig. 1b and Additional file 2: Figure S1). 
a
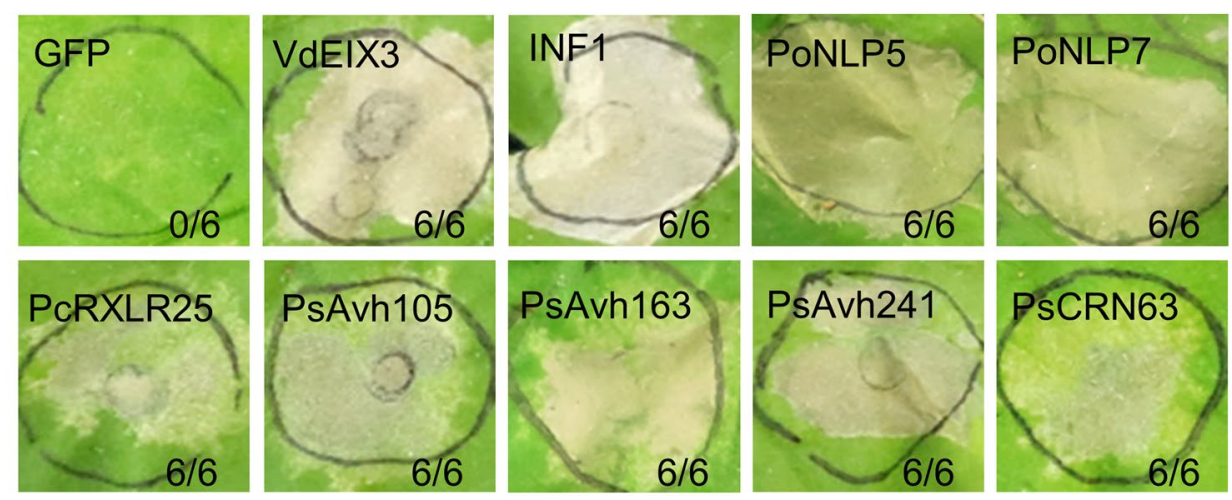

b
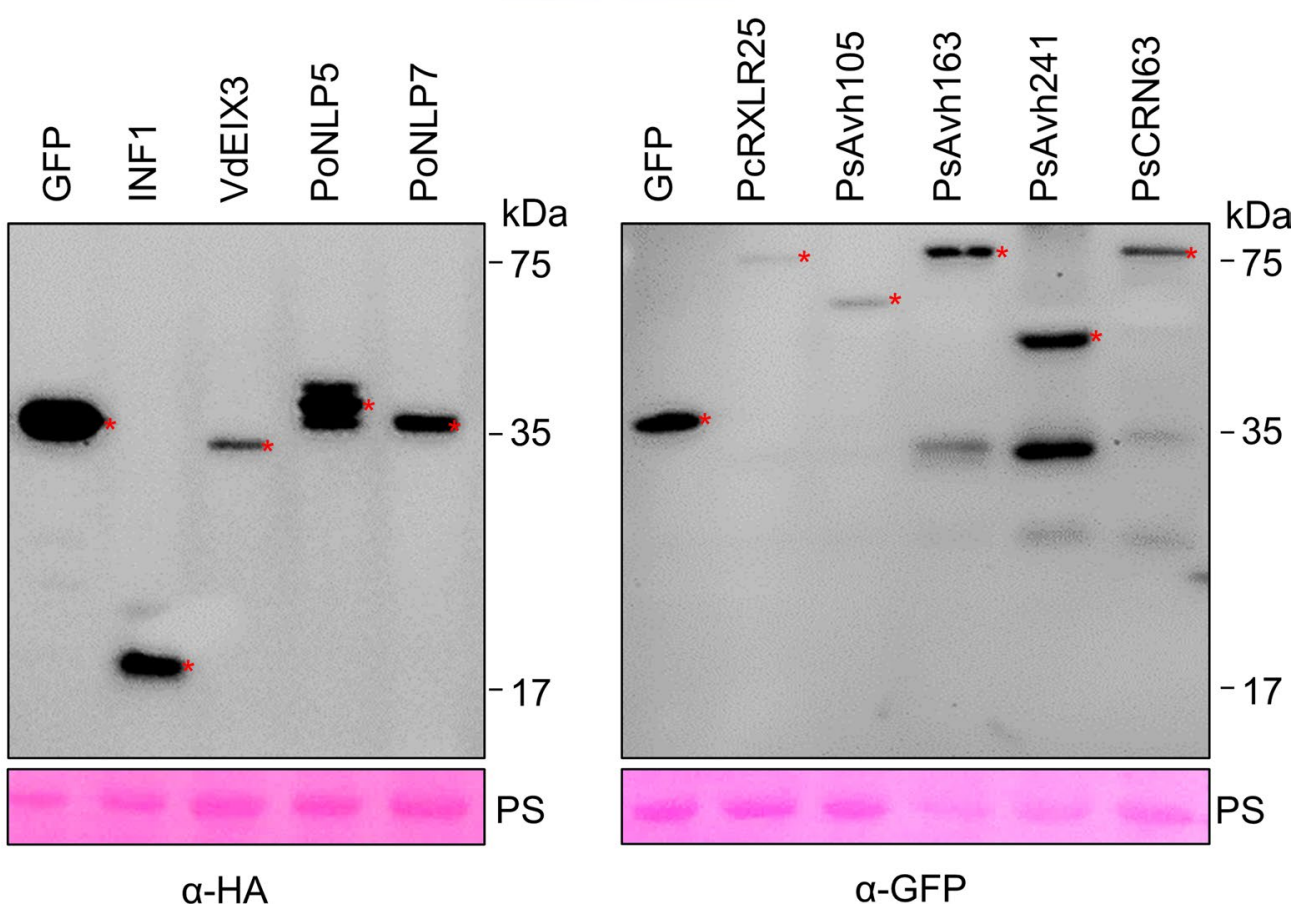

Fig. 1 Eight oomycete effectors cause HR-like phenotype in N. benthamiana. a Ectopic expression of eight effectors in N. benthamiana. Transient expression of VdEIX3, INF1, PoNLP5, PoNLP7, PCRXLR25, PsAvh163, PsAvh241 and PsCRN63 in N. benthamiana. GFP was used as a negative control and VdEIX 3 was used as a positive control. Photographs were taken at 6 days post-infiltration (dpi). Numbers represent the number of leaves showing cell death out of the number of leaves analyzed. $\mathbf{b}$ Western blot detection of eight oomycete effectors in $N$. benthamiana. Western blot analysis of indicated proteins expressed in N. benthamiana leaves. Leaves were collected after $36 \mathrm{~h}$ post-infiltration (hpi). Total protein loading was confirmed by Ponceau S staining

VdEIX3, INF1, PcRXLR25 and PsAvh163 induce SGT-dependent HR, while INF1, PcRXLR25, PsAvh163, PsAvh241 and PsCRN63 cause HSP90-dependent HR

SGT1 and HSP90 jointly regulate the stability and accumulation of NLR protein in plants. Many NLR proteins require the complex of ubiquitin ligase-related protein SGT1 and the heat shock protein HSP90 to activate cell death (Bos et al. 2006; Li et al. 2015). To test whether the cell death triggered by the selected effectors depends on NbSGT1 or NbHSP90, we individually silenced the two genes by VIGS. Compared with $T R V$-GUS control leaves, the expression levels of NbSGT1 and NbHSP9O were reduced $~ 90 \%$ and $~ 70 \%$ in indicated silenced leaves, respectively (Additional file 2: Figure S2). NbSGT1- and NbHSP90-silenced plants showed impaired growth phenotype (Additional file 2: Figure S3). Furthermore, INF1 induced cell death was abolished in SGT1- and HSP90-slienced plants (Fig. 2a). These results are similar to the previous report on SGT1- and HSP90-slienced N. benthamiana (Bos et al. 2006), indicating that NbSGT1 and NbHSP90 were silenced successfully. 


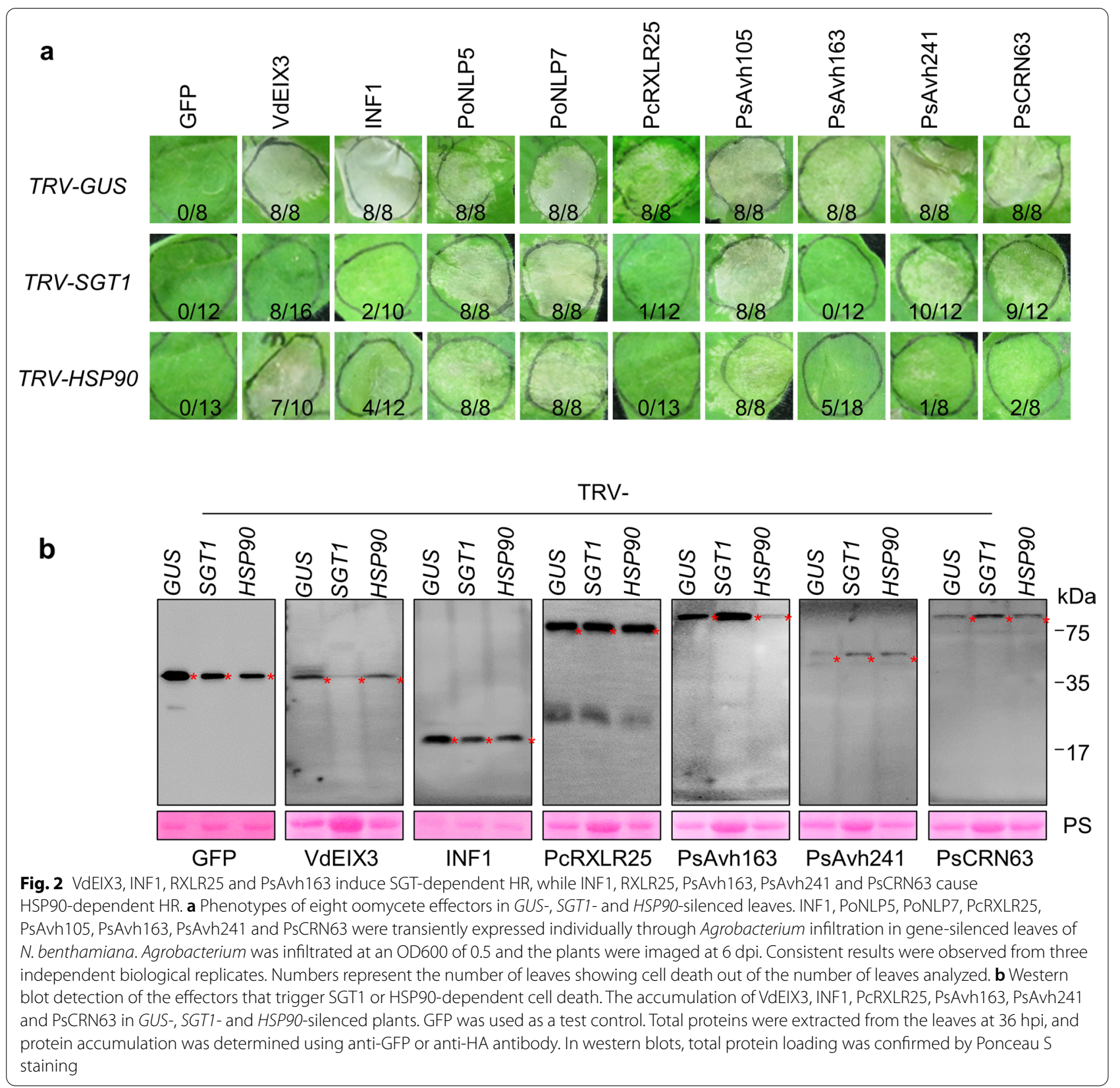

Silencing NbSGT1 abolished VdEIX3-, INF1-, PcRXLR25- and PsAvh163-triggered cell death in $N$. benthamiana, while silencing NbHSP90 abolished INF1-, PcRXLR25-, PsAvh163-, PsAvh241- and PsCRN63-triggered cell death (Fig. 2a). It was reported that protein accumulation might be affected in SGT1-VIGS plants (Fig. 2b) (Yu et al. 2019). In our western blot assay, all proteins did accumulate correctly in GUS-, SGT1- and HSP90-silenced lines (Fig. 2b). Based on these results, we speculated that these effectors-induced cell deaths might be related to the ETI pathway.
The cell death caused by eight effectors in $N$. benthamiana does not depend on NbNDR1 and NbEDS1

NDR1 and EDS1 are vital components of the ETI pathway (Aarts et al. 1998; McDowell et al. 2000; Day et al. 2006). We next tested whether the selected effector-triggered cell death is NDR1- or EDS1-dependent. As shown in Fig. 3, silencing NDR1 or EDS1 led to no effect on the cell death induced by all eight effectors. NDR1 and EDS1 expression levels were reduced $\sim 90 \%$ in the indicated gene-silenced leaves (Fig. 3a). Agrobacterium infiltration with any one of the eight effectors on EDS1- and NDR1-silenced leaves led 


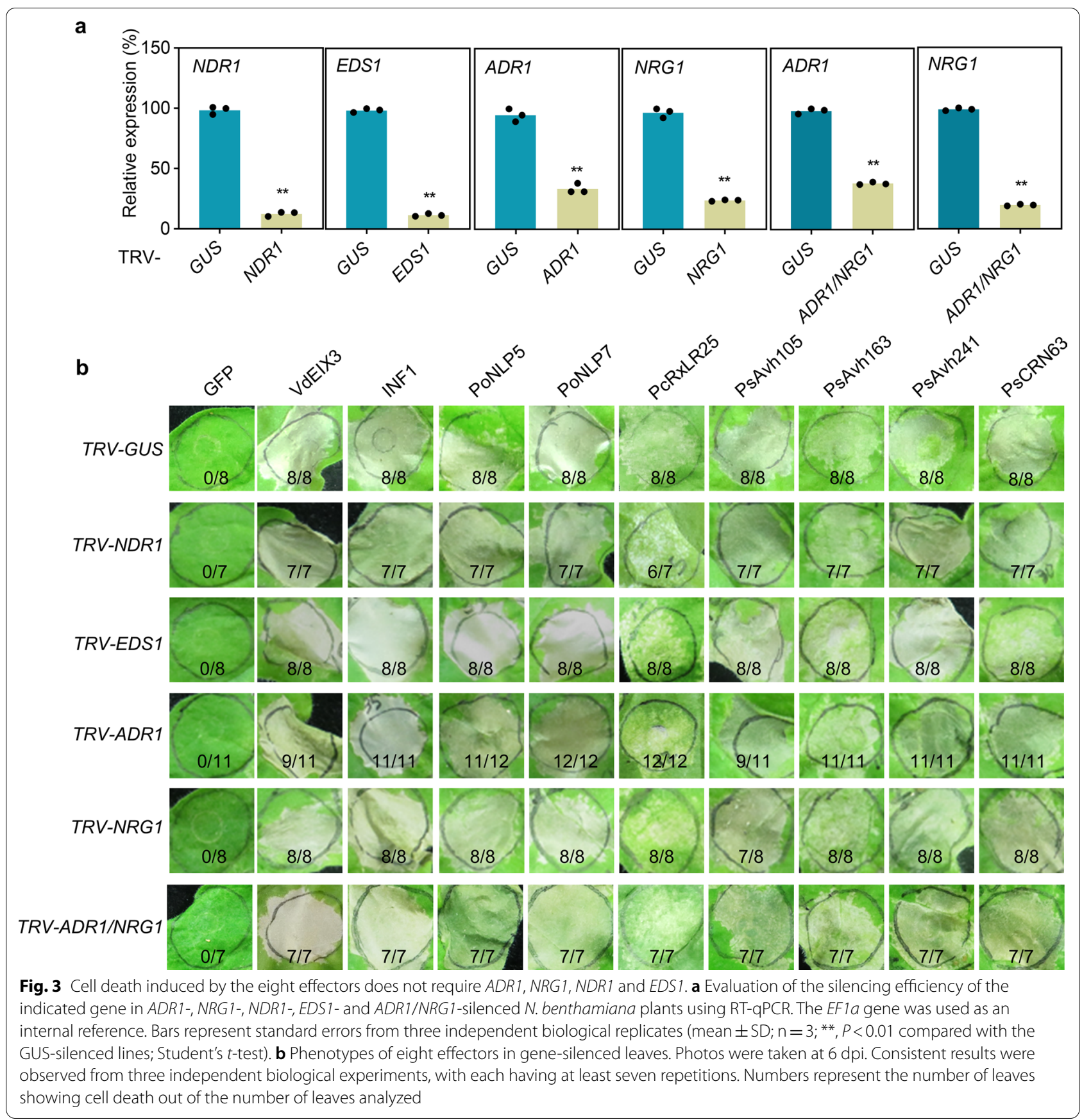

to the same cell death phenotype as those on GUS-silenced leaves (Fig. 3b), indicating that these effectors triggered EDS1- and NDR1-independent cell death.

\section{The cell death caused by eight effectors in $N$. benthamiana does not depend on RPW8-type helper NLR NbADR1 and NbNRG1}

NRG1 and ADR1 are RPW8-containing helper NLRs that are required for cell death triggered by recognition between AVR proteins and sensor NLRs (Castel et al. 2019; Wu et al. 2019). It was reported that NRG1 is a EDS1 downstream factor to regulate TIR-type sensor NLRs (TNLs)-mediated immunity in N. benthamiana (Qi et al. 2018). Besides, ADR1 is required for normal function of many TNLs (Wu et al. 2019). NRG1- and ADR1silenced lines were obtained and the eight effectors were individually expressed in the leaves (Fig. 3a). The expression levels of $A D R 1$ and NRG1 were both down-regulated 
by more than $60 \%$, indicating that the silencing efficiency is acceptable (Fig. 3a). The eight effectors still induced cell death in infiltration sites (Fig. 3b), which indicated that the cell death induced by the eight effectors did not require NRG1 or ADR1.

There is function redundancy between ADR1 and NRG1 (Saile et al. 2020). We thus silenced ADR1 and NRG1 simultaneously and found knock-down of both ADR1 and NRG1 did not abolish the cell death phenotype triggered by these effectors (Fig. 3a, b).

\section{PcRXLR25- and PsAvh163-triggered cell death in N. benthamiana depends on NRC helpers}

Except for RPW8-type helper NLRs, $N$. benthamiana has another significant type of helper NLRs, NRCs (NRC2, NRC3 and NRC4), which were reported to participate in defense responses in oomycetes, bacteria, viruses and nematodes (Wu et al. 2016; Derevnina et al. 2021). NbNRC4 is indispensable for Rpi-blb2 to recognize the $P$. infestans effector AVRblb2 in $N$. benthamiana (Wu et al. 2017). NRC2 and NRC3 are required for Pto-induced HR (Wu et al. 2016), while Bs2 and Sw5b trigger NRC2/3/4-dependent cell death in N.benthamiana (Wu et al. 2017). To figure out whether the NRC helpers have a role in the cell death caused by these eight effectors in $N$. benthamiana, we silenced NRC2, NRC3 and NRC4 separately and then expressed the eight effectors individually (Fig. 4a). The silencing efficiency of the three genes was confirmed and the eight effectors still triggered cell death in the silenced leaves (Fig. 4a, b).

However, when all the three NRCs were knocked down, the cell death induced by PcRXLR25 from Phytophthora capsici and PsAvh163 from P. sojae was totally inhibited (Fig. 5a, b). Western blot assay indicated that all the proteins accumulated normally in the silenced leaves (Fig. 5c). These results showed that PcRXLR25 and PsAvh163 triggered an NRCs-dependent cell death, indicating that PcRxLR25- and PsAvh163-triggered cell death in $N$. benthamiana was related to the ETI pathway. a

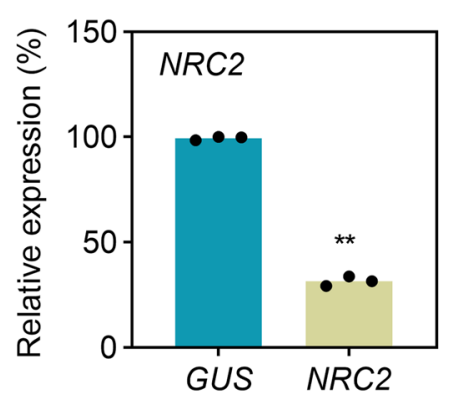

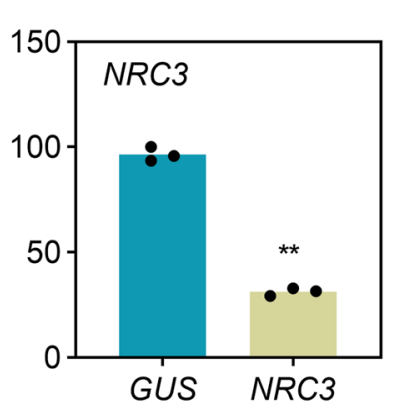

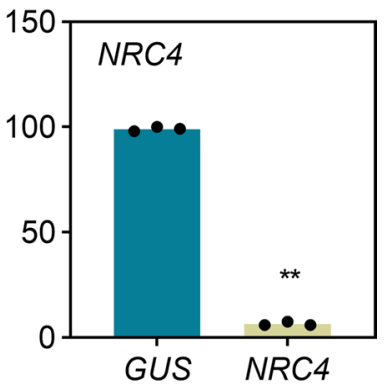

b

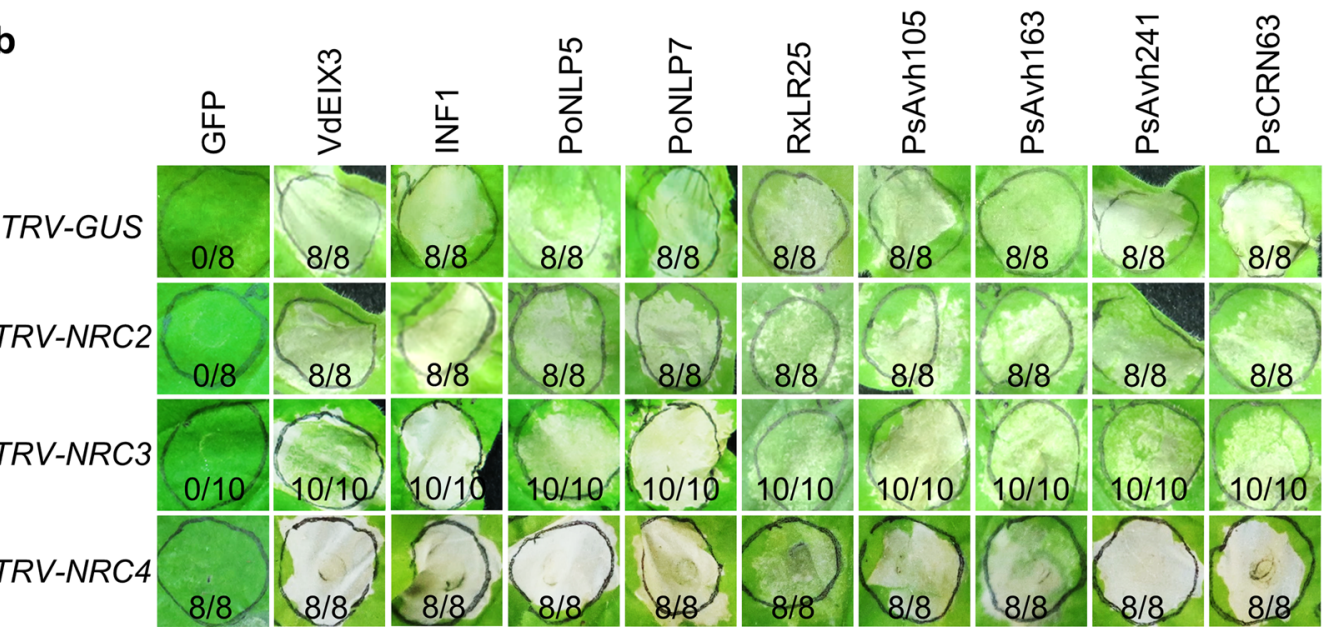

Fig. 4 Silencing individual NRC helper genes has no effect on the cell death triggered by the eight effectors in N. benthamiana. a Silencing efficiency of NbNRC2, NbNRC3 and NbNRC4 in the indicated gene knock-down N. benthamiana plants. Values represent average $\pm \operatorname{SE}(n=3) ;{ }^{* *}$, $P<0.01$ compared with the GUS; Student's t-test. b Transient expression of effectors in NRC2-, NRC3-, NRC4-silenced plants. Photographs were taken at $6 \mathrm{dpi}$. Consistent results were observed from three independent biological experiments, with each having at least eight repetitions. Numbers represent the number of leaves showing cell death out of the number of leaves analyzed 

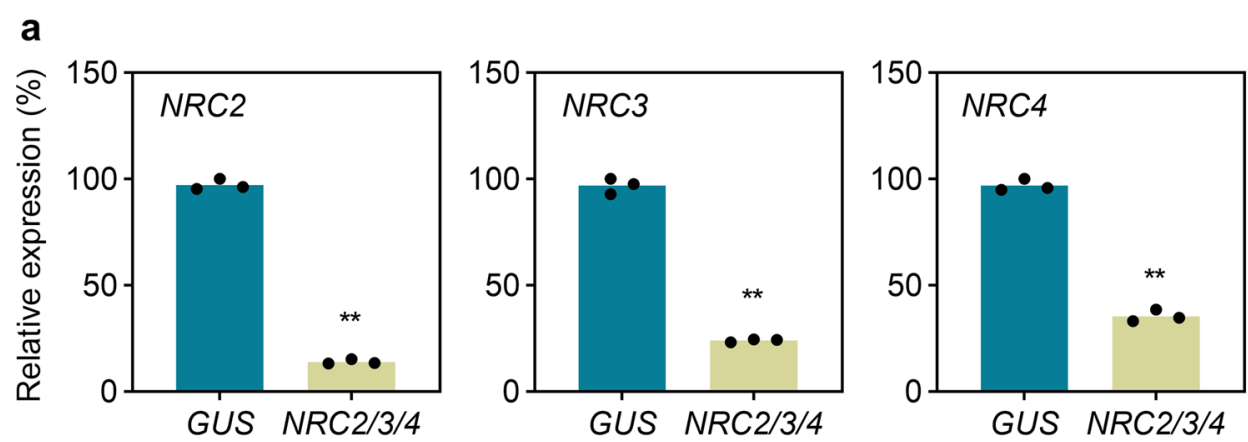

b

C
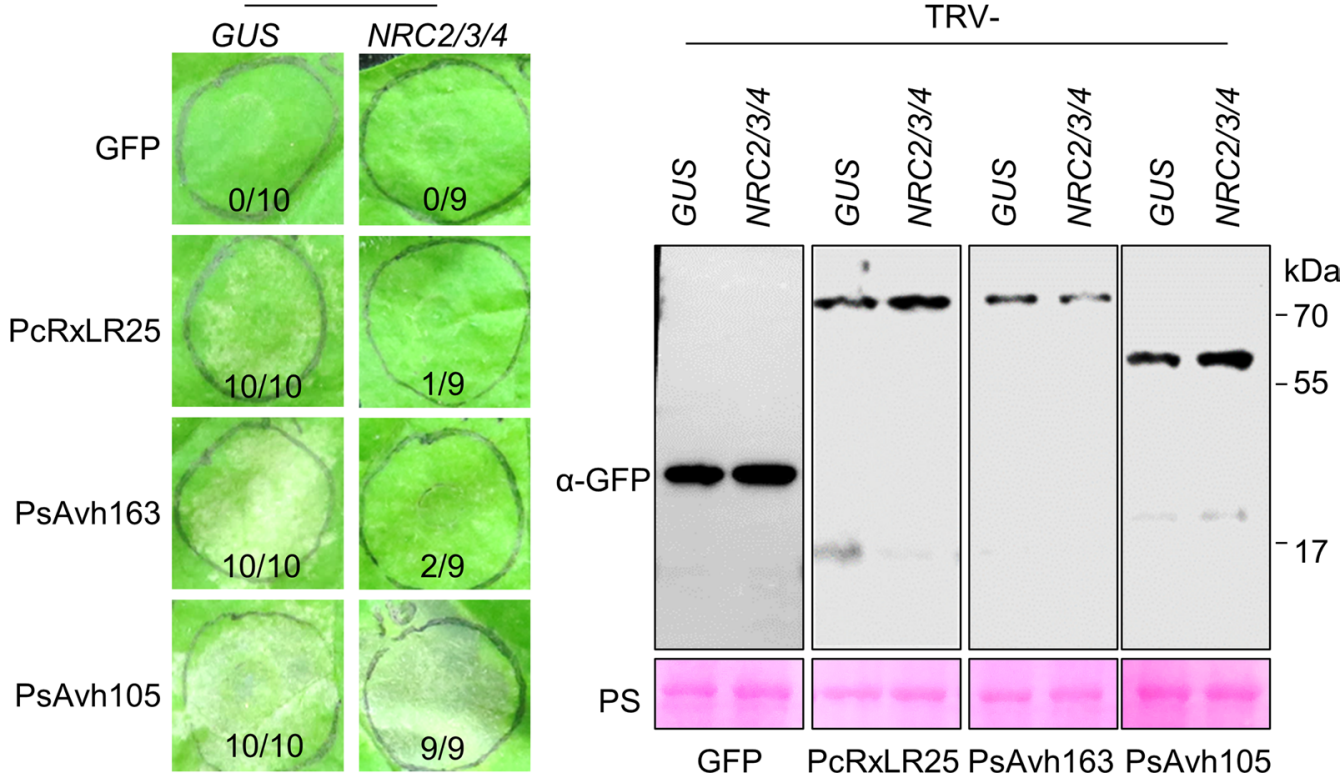

Fig. 5 Silencing NbNRC2, NbNRC3 and NbNRC4 simultaneously attenuates the cell death triggered by PCRXLR25 and PsAvh163 in N. benthamiana. a Silencing efficiency of NbNRC2, NbNRC3 and NbNRC4. Values represented average $\pm \mathrm{SE}(\mathrm{n}=3) ;{ }^{* *}, P<0.01$ compared with the GUS; Student's t-test. b Transient expression of GFP, PCRXLR25, PsAvh163 and PsAvh105 in GUS- and NRC2/3/4-silenced plants. GFP was used as a negative control. Consistent results were observed from three independent biological experiments. Numbers represent the number of leaves showing cell death out of the number of leaves analyzed. c The accumulation of GFP, PCRxLR25, PsAvh163 and PsAvh105 in GUS- and NRC2/3/4-silenced plants. GFP was used as a negative control. Total protein loading was confirmed by Ponceau S staining

\section{Cell death triggered by auto-activated NbNRC2/3/4 is dependent on SGT1 and HSP90}

According to the above experimental results, we concluded that HR induced by PcRXLR25 and PsAvh163 relied on SGT1/HSP90 and $\mathrm{NRC} 2 / 3 / 4$. It is interesting to figure out whether the correct functions of NRC2/3/4 require SGT1 and HSP90. The HSP90-SGT1 chaperone complex interacts with NLRs and plays a critical role in maintaining the stability of NLR proteins (such as Rx, RPS2, RPS5) and NLR-mediated resistance, (Takahashi et al. 2003; Holt et al. 2005; Botër et al. 2007). Meanwhile, this chaperone complex contributes to the hybrid incompatibility in Nicotiana (Katsuyama et al. 2021). To investigate the functional relationship between NRCs and SGT1 or HSP90, NRCs auto-activated mutant vector $\left(\mathrm{NRC} 2^{\mathrm{H} 480 \mathrm{R}}, \mathrm{NRC} 3^{\mathrm{D} 480 \mathrm{~V}}\right.$, NRC4 ${ }^{\text {D478V }}$ ) was constructed (Derevnina et al. 2021). As shown in Fig. 6a, NRC2 ${ }^{\mathrm{H} 480 \mathrm{R}}, \mathrm{NRC} 3^{\mathrm{D} 480 \mathrm{~V}}$ and $\mathrm{NRC} 4^{\mathrm{D} 478 \mathrm{~V}}$ induced cell death in control leaves, which confirmed that $\mathrm{NRC} 2^{\mathrm{H} 480 \mathrm{R}}, \mathrm{NRC} 3^{\mathrm{D} 480 \mathrm{~V}}$ and $\mathrm{NRC} 4^{\mathrm{D} 478 \mathrm{~V}}$ are auto-activated mutants. However, all the auto-activated mutants failed to induce cell death in SGT1- and HSP90-silenced plants (Fig. 6a), indicating that the correct function of $\mathrm{NRC2/3/4}$ required both SGT1 and HSP90.

We next tested the interaction of $\mathrm{NRC} 2 / 3 / 4$ with SGT1 and HSP90 using a luciferase complementation assay. NRC2/3/4 were fused with nLUC in their 
a

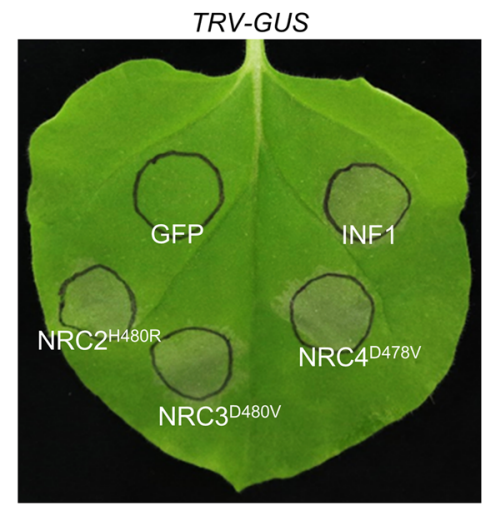

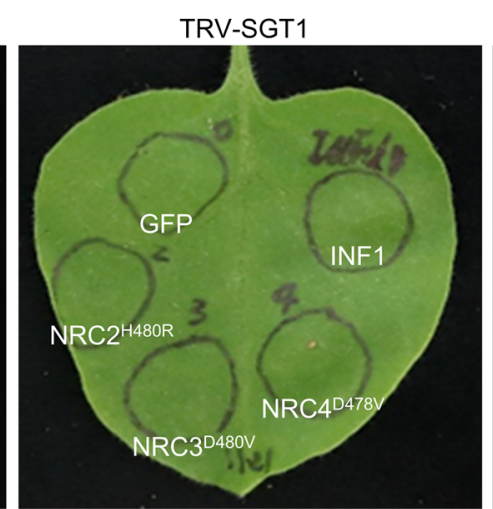
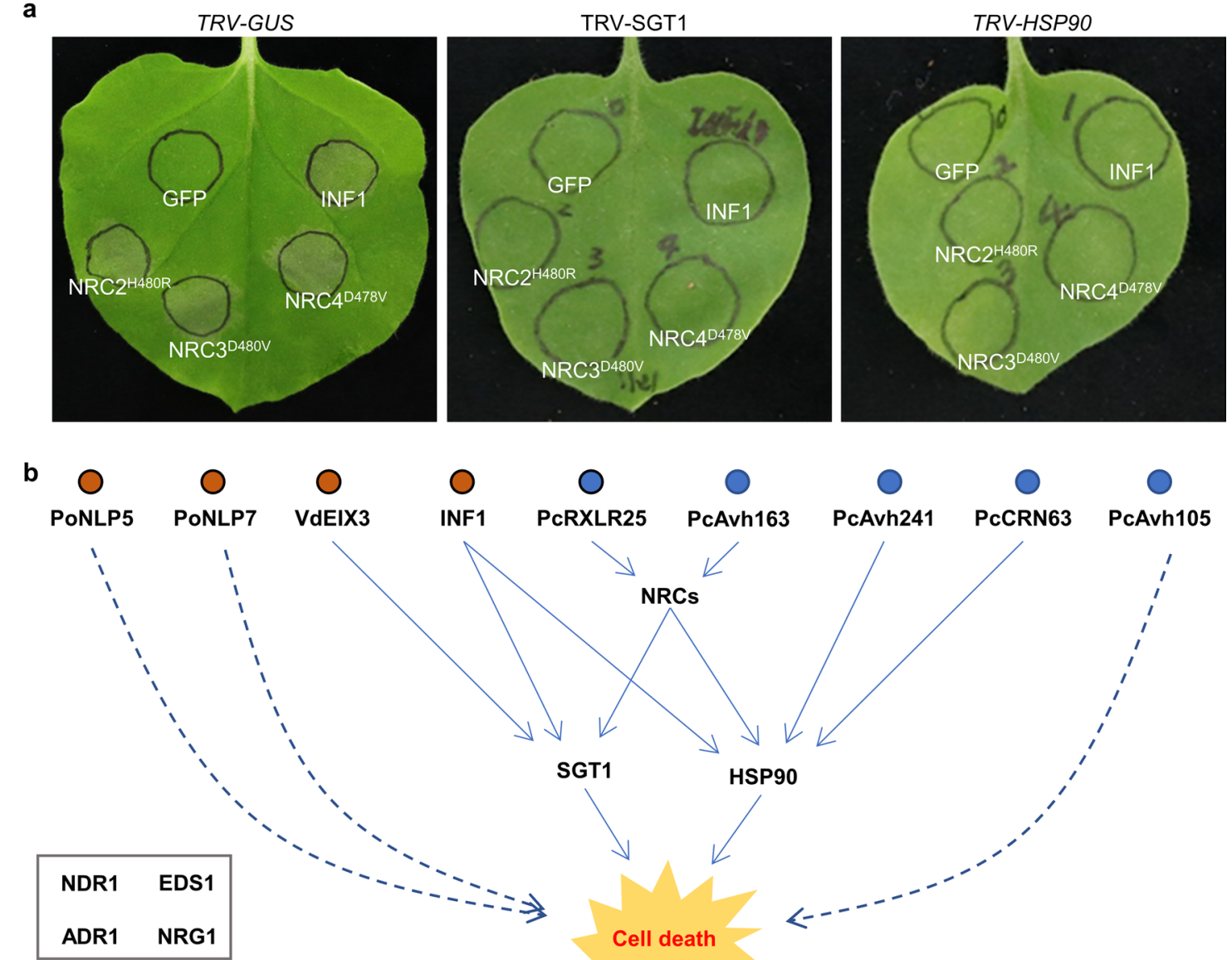

Unnecessary components

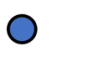

PcAvh105
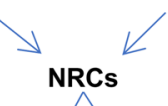

$\measuredangle$
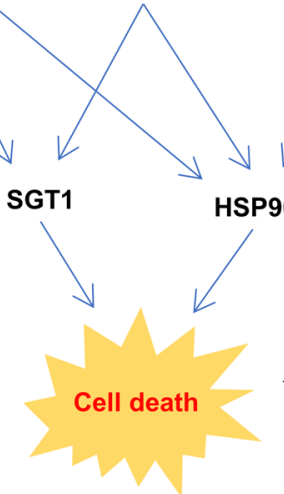

SP90

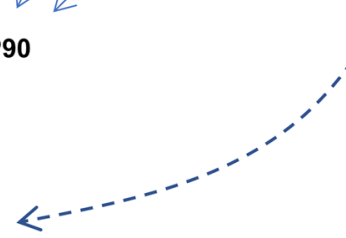

cytoplasmic effectors

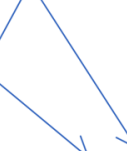

\section{apoplastic effectors}

Fig. 6 Correct function of NRC2/3/4 requires SGT1 and HSP90. a The phenotypes of auto-activated NbNRC2, NbNRC3 and NbNRC4 in GUS-, SGT1- and HSP90-silenced N. benthamiana plants. Photographs were taken at $2 \mathrm{dpi}$. Numbers represent the number of leaves showing cell death out of the number of leaves analyzed. $\mathbf{b}$ Oomycete effectors-induced cell death is differentially dependent on effector-triggered immunity pathway components. Dotted arrows indicate that host genes required for the effector-induced cell death is unknown

C terminal and co-expressed with SGT1 or HSP90 fused with cLUC in $\mathrm{N}$ terminal in leaves. The combination of FLS2 (flagellin-sensitive2) and G $\beta$ (G protein $\beta$-subunit1) from Arabidopsis thaliana, a well-known associated proteins (Liang et al. 2016), were used as positive control. No chemiluminescence signal could be detected in the infiltration sites where the combination of NRCs and HSP90/SGT1 was co-expressed, while positive control (FLS2-nLUC and cLUC-G $\beta$ ) displayed strong signals (Additional file 2: Figure S4). This indicated that NRC2/3/4 and SGT1/HSP90 may not interact with each other in our system.

\section{Discussion}

Many cytoplasmic effectors secreted by oomycete pathogens could induce cell death in plants. According to the results from two large-scale screenings of RXLR effectors, nearly 6-7\% RXLR effectors from $P$. sojae and $P$. capsici could trigger cell death in $N$. benthamiana ( $\mathrm{Li}$ et al. 2019; Wang et al. 2011). Likewise, RXLR effectors from Pythium species are able to cause cell death in plants (Ai et al. 2020). Same situation can be applied to CRN effectors (Stam et al. 2013). These cell deathinduced effectors may be toxic effectors capable of killing cells directly. Alternatively, some of them may be AVR 
proteins recognized by $\mathrm{R}$ proteins and trigger $\mathrm{HR}$. In this study, we identified two effectors, both of which are RXLR effectors and have potential to be AVR candidates.

Ectopic expression of some CRN effectors from oomycetes and fungi leads to severe cell death in plant, insect and animal cells (Schornack et al. 2010; Stam et al. 2013; Ramirez-Garces et al. 2016; Shen et al. 2019). In this study, no CRN effector was selected as an AVR protein candidate. The screening scale of this study is not broad enough, so whether there is a chance for CRN effectors to be AVR proteins is still unknown. In many circumstances, CRN effector-induced cell death requires nuclear localization of CRN effectors (Schornack et al. 2010; Stam et al. 2013). Similarly, some NLR proteins need nucleus localization for correct functions (Shen et al. 2007; Xu et al. 2014). It is needed to figure out whether there is an $R$ gene that can recognize CRN effectors.

Apoplastic effectors such as NLP effectors are toxic effectors that bind to plant-specific glycosylinositol phosphorylceramide (GIPC) sphingolipids to kill plant cells (Lenarcic et al. 2017). As expected, PoNLP5- and PoNLP7-triggered cell death did not require any of the ETI components tested in this study. Interestingly, VdEIX3, an effector to induce PTI, also relied on SGT1 to produce cell death. However, it did not depend on other ETI components like EDS1 or helper NLRs. Such phenomenon may be explained by the recently uncovered tight association between ETI and PTI (Ngou et al. 2021). Similarly, another PTI-related apoplastic effector, INF1, also required SGT1 and HSP90 to induce cell death.

HR mediated by PcRXLR25 and PsAvh163 was dependent on Solanaceae-specific helper NLR, NRC2/3/4, but not EDS1 or other types of helper NLR, which indicated that the sensor NLR of PcRXLR25 and PsAvh163 might be CNL-type $R$ genes. In addition, PcRXLR25- and PsAvh163-induced HR depended on NRC2/3/4, SGT1 and HSP90 simultaneously. This inspired us to test the association of NRC2/3/4 with SGT1 and HSP90, and the luciferase complementation assay showed that no interaction was found between them. However, since the interaction between NLR and other proteins is a complicated issue, we can only conclude that no interaction was observed in our system. Surprisingly, we detected that the auto-activated NRC2/3/4 required SGT1 and HSP90 to induce HR. The SGT1 and HSP90 complex is crucial in stabilizing NLR-type sensors (Shirasu 2009). As a result of our study, SGT1 and HSP90 complex may also help to stabilize helper NLRs.

According to the iceberg model, the vast majority of AVR/R pairs are hidden (Thordal-Christensen 2020). It is a promising way to reveal the hidden AVR proteins via investigating the requirement of cell death-induced effector for ETI signaling components. By screening effectors in gene-silenced tobacco leaves, we have successfully identified two putative AVR proteins, PcRXLR25 and PsAvh163. In future studies, the virulent function of these two effectors should be investigated and their cognate $\mathrm{R}$ proteins should be identified. Furthermore, we have found that SGT1 and HSP90 are required for normal function of NRC2/3/4. Next, we need to determine how SGT1 and HSP90 regulate NRC2/3/4.

\section{Conclusions}

In this study, we screened eight cell death-induced effectors from oomycete pathogens in $N$. benthamiana to figure out which one is associated with ETI (Fig. 6b). The cell death phenotypes caused by these effectors were tested in the absence of vital ETI signaling components. As a result, we found that PsAvh163, VdEIX3 and PcRXLR25 require SGT1 to induce cell death, while silencing NbHSP90 abolishes PcRXLR25-, PsAvh163-, PsAvh241- and PsCRN63-triggered cell death. None of the eight effectors induce cell death through EDS1, NDR1, NRG1 and ADR1. HR induced by PcRXLR25 and PsAvh163 requires NRC2/3/4, suggesting that these two effectors are related to ETI. Hence, they are probably AVR protein candidates. Finally, we found that auto-activated NRC2/3/4 also relies on both SGT1 and HSP90 to generate $\mathrm{HR}$.

\section{Methods \\ Plasmid construct}

The effectors used in the study are all existing vectors in the laboratory. Briefly, GFP (as a control), INF1 (as a control), VdEIX3, PoNLP5, PoNLP7 were cloned into pBin3xHA. PcRXLR25, PsAvh105, PsAvh163, PsAvh24 and PsCRN63 (without signal peptide) were cloned into pBinGFP2. NRC2, NRC3, NRC4, SGT1 and HSP90 were amplified from $N$. benthamiana. For luciferase complementation assay, NRC2, NRC3 and NRC4 were cloned into pCAMBIA1300-35S-HA-Nluc-RBS, SGT1 and HSP90 were cloned into pCAMBIA1300-35S-Cluc-RBS. To overexpress auto-activated $\mathrm{NbNRC} 2 / 3 / 4$ in $N$. benthamiana, $\mathrm{NRC} 2^{\mathrm{H} 480 \mathrm{R}}, \mathrm{NRC} 3^{\mathrm{D} 480 \mathrm{~V}}$ and $\mathrm{NRC} 4^{\mathrm{D} 478 \mathrm{~V}}$ were constructed in pBin3xHA. In order to silence $S G T 1, H S P 90$, EDS1, NDR1, ADR1, NRG1, NRC2, NRC3 or NRC4 in N. benthamiana, the reported gene fragment was inserted into pTRV2 vector individually (Burch-Smith et al. 2004). Primers are listed in the Additional file 1: Table S2.

\section{Plant growth conditions}

$N$. benthamiana were grown in greenhouse at $25^{\circ} \mathrm{C}$ with $60 \%$ relative humidity and a $16-\mathrm{h}$ light/8-h dark photoperiod. VIGS-treated $N$. benthamiana plants used in this study were grown in the greenhouse at a temperature of 
$22{ }^{\circ} \mathrm{C}$ under a 16 -h light/8-h dark photoperiod and 58\% relative humidity.

\section{Transient expression and VIGS in N. benthamiana}

The indicated recombinant constructs were transformed into Agrobacterium tumefaciens strain GV3101. For infiltration, Agrobacterium strains were cultured at $28{ }^{\circ} \mathrm{C}$ and $220 \mathrm{rpm}$ for $48 \mathrm{~h}$, and the cells were collected after centrifugation at $4000 \mathrm{rpm}$ for $4 \mathrm{~min}$. The cells were washed and then re-suspended in infiltration medium $[10 \mathrm{mM}$ $\mathrm{MgCl}_{2}, 10 \mathrm{mM}$ MES (PH5.7) and $200 \mu \mathrm{M}$ acetosyringone] to an appropriate optical density (OD) at $600 \mathrm{~nm}$ $(0.4-0.6)$. Five-week-old $N$. benthamiana leaves were infiltrated for transient expression.

For Agrobacterium-mediated VIGS, TRV vectors pTRV-RNA1 and pTRV-RNA2, namely pTRV-SGT1, pTRV-HSP90, pTRV-EDS1, pTRV-NDR1, pTRV-ADR1, pTRV-NRG1, pTRV-NRC2, pTRV-NRC3, pTRV-NRC4, pTRV-ADR1/NRG1, pTRV-NRC2/3/4, pTRV-GUS (negative control), and pTRV-PDS (positive control), were introduced into A. tumefaciens strain GV3101 by electroporation. Agrobacterium suspensions containing pTRV-RNA1 and pTRV-RNA2 derivatives were mixed at an equal ratio and inoculated into the true leaves of 20-day-old soil-grown $N$. benthamiana. Treated $N$. benthamiana plants were maintained at $22{ }^{\circ} \mathrm{C}$ under a 16-h light/8-h dark photoperiod for 25 days before Agrobacterium transient expression.

\section{RT-qPCR analysis and bioinformatics analysis}

For RT-qPCR analysis, total RNA was extracted from $N$. benthamiana leaves with an RNA-simple Total RNA Kit (Tiangen Biotech Co., Ltd., Beijing, China) according to the operating instructions. $N$. benthamiana cDNA was synthesized with the HiScript II Q RT SuperMix for qPCR (Vazyme Biotech Co., Ltd., Nanjing, China). Realtime PCR was performed by using a SYBR Premix Ex Taq Kit (Takara Bio Inc., Shiga, Japan) on an ABI Prism 7500 Fast Real-Time PCR system following the instructions. Gene expression levels were normalized to the expression of $N b E F 1 a$, a stably expressed reference gene in $N$. benthamiana. The primers used are listed in Additional file 1: Table S2. All the RT-qPCR results shown in this study were calculated from three independent biological replicates.

\section{Western blotting}

To extract proteins from $N$. benthamiana, leaves were frozen in liquid nitrogen and polished to a fine powder. For normal western blot assay, extraction buffer $(50 \mathrm{mM}$ HEPES, $150 \mathrm{mM} \mathrm{KCL}, 1 \mathrm{mM}$ EDTA, and 0.1\% Triton $\mathrm{X}-100 ; \mathrm{pH}$ 7.5), supplemented with $1 \mathrm{mM}$ DTT and protease inhibitor cocktail (Sigma-Aldrich, St. Louis, $\mathrm{MO}$, USA), was used for protein extraction from plant materials. Anti-HA (1:5, 000; \#M20013; Abmart Inc., Shanghai, China), anti-GFP (1:5, 000; \#M20004; Abmart), antibodies were used to bind the protein with the corresponding tag.

\section{Luciferase complementation assay}

The coding sequence of indicated genes was cloned into pCAMBIA1300-35S-HA-Nluc-RBS or pCAMBIA130035S-Cluc-RBS and then was transferred into A. tumefaciens strain GV3101. Agrobacterium strains carrying the indicated Cluc and Nluc constructs were infiltrated into $N$. benthamiana leaves. Leaves were sprayed with $1 \mathrm{mM}$ luciferin (Biovision) and luminescence was detected with a microplate reader (BioTek, Beijing, China) after $15 \mathrm{~min}$.

\section{Confocal microscopy}

The GFP-fused constructs were expressed in $N$. benthamiana leaves by Agrobacterium-mediated transient expression. The signal of fluorescence was imagined using a confocal microscope under a $488 \mathrm{~nm}$ excitation wavelength.

\section{Accession number}

The primary accession codes for INF1, VdEIX3, PoNLP5, PoNLP7, RXLR25, PsAvh105, PsAvh163, PsAvh241 and PsCRN63 that support the finding of this study were shown as AY830090.1 (INF1), VDAG_06165 (VdEIX3), PYOLI_00013111-RA (PoNLP5), PYOLI_00013113RA (PoNLP7), PHYCAscaffold_81:176234-177661 (RXLR25), Ps138565 (PsAvh105), Ps141933 (PsAvh163), Ps133912 (PsAvh241) and HQ231783.1 (PsCRN63), respectively.

\section{Abbreviations}

ADR1: Activated disease resistance 1; CRN: Crinkling- and necrosis-inducing protein; EDS1: Enhanced disease susceptibility 1; ETI: Effector-triggered immunity; HR: Hypersensitive response; NDR1: Nonrace-specific disease resistance 1; NLR: Nucleotide-binding leucine-rich repeat; NRC: NLR-required for cell death; NRG1: N requirement gene 1; PTI: Pattern-triggered immunity.

\section{Supplementary Information}

The online version contains supplementary material available at https://doi. org/10.1186/s42483-022-00109-1.

Additional file 1: Table S1. Eight effectors from oomycetes that trigger cell death in N. benthamiana. Table S2. Primers used in this study

Additional file 2: Figure S1. Observation of GFP-tagged effectors using confocal microscope. Confocal microscopy images were taken at $36 \mathrm{hpi}$. Scale bars $=50 \mu \mathrm{m}$. Figure S2. The silencing efficiency of NbSGT1 and NbHSP90. Transcript levels of SGT1 and HSP90 were analyzed by RT-qPCR. The EFla gene was used as an internal reference. Bars represent standard errors from three independent biological replicates (mean $\pm S D ; n=3$; ${ }^{*}$, $P<0.01$ compared with the GUS-silenced lines; Student's $t$-test). Figure 
S3. Phenotypes of gene-silenced $\mathrm{N}$. benthamiana. Photos were taken at 25 dpi. Figure S4. The luciferase complementation assay between NRCs and SGT1/HSP90. A luciferase complementation assay was performed on $N$. benthamiana plants by Agrobacterium-mediated transient expression of the indicated constructs. The combination of FLS2-nLUC + cLUC-GB was used as a positive control. This experiment was repeated three times independently with four replicates.

\section{Acknowledgements}

We appreciate Dr. Min Zhu and Prof. Xiaorong Tao at Nanjing Agricultural University for their gift of NRC3/4 auto-activated mutant, constructive suggestions and help. We appreciate Dr. Meixiang Zhang at Shaanxi Normal University for his help.

\section{Authors' contributions}

$\mathrm{DD}, \mathrm{XD}$ and $\mathrm{GA}$ conceived and designed the project, jointly performed data analysis and wrote the manuscript. XD, CX and WP performed the experiments. XD and GA analysed data. DD, XD, GA and ZY wrote and modified the manuscript. All authors read and approved the final manuscript.

\section{Funding}

The work was supported by the National Natural Science Foundation of China (31625023 and 32072507) and the Fundamental Research Funds for the Central Universities (KYT202001 and JCQY201904).

\section{Availability of data and materials}

Not applicable.

\section{Declarations}

Ethics approval and consent to participate

Not applicable.

\section{Consent for publication}

Not applicable.

\section{Competing interests}

The authors declare that they have no competing interests.

Received: 13 December 2021 Accepted: 28 January 2022

Published online: 10 February 2022

\section{References}

Aarts N, Metz M, Holub E, Staskawicz BJ, Daniels MJ, Parker JE. Different requirements for EDS1 and NDR1 by disease resistance genes define at least two $R$ gene-mediated signaling pathways in Arabidopsis. Proc Nat Acad Sci U S A. 1998;95(17):10306-11. https://doi.org/10.1073/pnas.95. 17.10306

Ai G, Yang K, Ye W, Tian Y, Du Y, Zhu H, et al. Prediction and characterization of RXLR effectors in Pythium species. Mol Plant-Microbe Interact. 2020;33(8):1046-58. https://doi.org/10.1094/MPMI-01-20-0010-R.

Ballvora A, Ercolano MR, Weiss J, Meksem K, Bormann CA, Oberhagemann $P$, et al. The $R 1$ gene for potato resistance to late blight (Phytophthora infestans) belongs to the leucine zipper/NBS/LRR class of plant resistance genes. Plant J. 2002;30(3):361-71. https://doi.org/10.1046/j.1365-313X. 2001.01292.x.

Bonardi V, Tang S, Stallmann A, Roberts M, Cherkis K, Dangl JL. Expanded functions for a family of plant intracellular immune receptors beyond specific recognition of pathogen effectors. Proc Natl Acad Sci USA. 2011;108(39):16463-8. https://doi.org/10.1073/pnas.1113726108.

Bos JIB, Kanneganti TD, Young C, Cakir C, Huitema E, Win J, et al. The C-terminal half of Phytophthora infestans RXLR effector AVR3a is sufficient to trigger R3a-mediated hypersensitivity and suppress INF1-induced cell death in Nicotiana benthamiana. Plant J. 2006;48(2):165-76. https://doi.org/10. 1111/j.1365-313X.2006.02866.X.
Botër M, Amigues B, Peart J, Breuer C, Kadota Y, Casais C, et al. Structural and functional analysis of SGT1 reveals that its interaction with HSP90 is required for the accumulation of $\mathrm{Rx}$, an $\mathrm{R}$ protein involved in plant immunity. Plant Cell. 2007;19(11):3791-804. https://doi.org/10.1105/tpc. 107.050427.

Burch-Smith TM, Anderson JC, Martin GB, Dinesh-Kumar SP. Applications and advantages of virus-induced gene silencing for gene function studies in plants. Plant J. 2004;39(5):734-46. https://doi.org/10.1111/j. 1365-313X.2004.02158.x.

Burki F, Shalchian-Tabrizi K, Pawlowski J. Phylogenomics reveals a new "megagroup" including most photosynthetic eukaryotes. Biol Lett. 2008;4(4):366-9. https://doi.org/10.1098/rsbl.2008.0224.

Castel B, Ngou PM, Cevik V, Redkar A, Kim DS, Yang Y, et al. Diverse NLR immune receptors activate defence via the RPW8-NLR NRG1. New Phytol. 2019;222(2):966-80. https://doi.org/10.1111/nph.15659.

Day B, Dahlbeck D, Staskawicz BJ. NDR1 interaction with RIN4 mediates the differential activation of multiple disease resistance pathways in Arabidopsis. Plant Cell. 2006;18(10):2782-91. https://doi.org/10.1105/ tpc. 106.044693.

Derevnina L, Contreras MP, Adachi H, Upson J, Cruces AV, Xie R, et al. Plant pathogens convergently evolved to counteract redundant nodes of an NLR immune receptor network. PLoS Biol. 2021;19(8): e3001136. https://doi.org/10.1371/journal.pbio.3001136.

Dodds PN, Rathjen JP. Plant immunity: towards an integrated view of plantpathogen interactions. Nat Rev Genet. 2010;11:539-48. https://doi.org/ $10.1038 /$ nrg2812.

Dongus JA, Parker JE. EDS1 signalling: at the nexus of intracellular and surface receptor immunity. Curr Opin Plant Biol. 2021;62: 102039. https:// doi.org/10.1016/J.Pbi.2021.102039.

Dou DL, Zhou JM. Phytopathogen effectors subverting host immunity: different foes, similar battleground. Cell Host Microbe. 2012;12(4):484-95. https://doi.org/10.1016/j.chom.2012.09.003.

Foster SJ, Park TH, Pel M, Brigneti G, Sliwka J, Jagger L, et al. Rpi-vnt1.1, a $\mathrm{Tm}$-2(2) homolog from Solanum venturii, confers resistance to potato late blight. Mol Plant-Microbe Interact. 2009;22(5):589-600. https://doi. org/10.1094/Mpmi-22-5-0589.

Gabriels SHEJ, Vossen JH, Ekengren SK, van Ooijen G, Abd-El-Haliem AM, van den Berg GCM, et al. An NB-LRR protein required for HR signalling mediated by both extra- and intracellular resistance proteins. Plant $\mathrm{J}$. 2007;50(1):14-28. https://doi.org/10.1111/j.1365-313X.2007.03027.x.

Holt BF, Belkhadir Y, Dangl JL. Antagonistic control of disease resistance protein stability in the plant immune system. Science. 2005;309(5736):929-32. https://doi.org/10.1126/science.1109977.

Huang SW, van der Vossen EAG, Kuang HH, Vleeshouwers VGAA, Zhang NW, Borm TJA, et al. Comparative genomics enabled the isolation of the R3a late blight resistance gene in potato. Plant J. 2005;42(2):251-61. https://doi.org/10.1111/j.1365-313X.2005.02365.X.

Jones JDG, Dangl JL. The plant immune system. Nature. 2006;444(7117):323-9. https://doi.org/10.1038/nature05286.

Jubic LM, Saile S, Furzer OJ, El Kasmi F, Dangl JL. Help wanted: helper NLRS and plant immune responses. Curr Opin Plant Biol. 2019;50:82-94. https://doi.org/10.1016/j.pbi.2019.03.013.

Kamoun S, Furzer O, Jones JDG, Judelson HS, Ali GS, Dalio RJD, et al. The Top 10 oomycete pathogens in molecular plant pathology. Mol Plant Pathol. 2015:16(4):413-34. https://doi.org/10.1111/mpp.12190.

Katsuyama Y, Doi M, Shioya S, Hane S, Yoshioka M, Date S, et al. The role of chaperone complex HSP90-SGT1-RAR1 as the associated machinery for hybrid inviability between Nicotiana gossei Domin and N. tabacum L. Gene. 2021;776: 145443. https://doi.org/10.1016/j.gene.2021.145443.

Knepper C, Savory EA, Day B. Arabidopsis NDR1 is an integrin-like protein with a role in fluid loss and plasma membrane-cell wall adhesion. Plant Physiol. 2011a;156(1):286-300. https://doi.org/10.1104/pp.110.169656.

Knepper C, Savory EA, Day B. The role of NDR1 in pathogen perception and plant defense signaling. Plant Signaling Behav. 2011b;6(8):1114-6. https://doi.org/10.4161/psb.6.8.15843.

Lenarcic T, Albert I, Bohm H, Hodnik V, Pirc K, Zavec AB, et al. Eudicot plantspecific sphingolipids determine host selectivity of microbial NLP cytolysins. Science. 2017;358(6369):1431. https://doi.org/10.1126/scien ce.aan6874.

Li Q, Ai G, Shen D, Zou F, Wang J, Bai T, et al. A Phytophthora capsici effector targets ACD11 binding partners that regulate ROS-mediated defense 
response in Arabidopsis. Mol Plant. 2019;12(4):565-81. https://doi.org/ 10.1016/j.molp.2019.01.018.

Li X, Kapos P, Zhang Y. NLRs in plants. Curr Opin Immunol. 2015;32:114-21. https://doi.org/10.1016/j.coi.2015.01.014.

Liang X, Ding P, Lian K, Wang J, Ma M, Li L, et al. Arabidopsis heterotrimeric G proteins regulate immunity by directly coupling to the FLS2 receptor. Elife. 2016;5: e13568. https://doi.org/10.7554/eLife.13568.

McDowell JM, Cuzick A, Can C, Beynon J, Dangl JL, Holub EB. Downy mildew (Peronospora parasitica) resistance genes in Arabidopsis vary in functional requirements for NDR1, EDS1, NPR1 and salicylic acid accumulation. Plant J. 2000;22(6):523-9. https://doi.org/10.1046/j.1365-313x.2000.00771.x.

McNeece BT, Pant SR, Sharma K, Niruala P, Lawrence GW, Klink VP. A glycine max homolog of NON-RACE SPECIFIC DISEASE RESISTANCE 1 (NDR1) alters defense gene expression while functioning during a resistance response to different root pathogens in different genetic backgrounds. Plant Physiol Biochem. 2017;114:60-71. https://doi.org/10.1016/j.plaphy. 2017.02.022.

Ngou BPM, Jones JDG, Ding P. Plant immune networks. Trends Plant Sci. 2021. https://doi.org/10.1016/j.tplants.2021.08.012.

Pais M, Win J, Yoshida K, Etherington GJ, Cano LM, Raffaele S, et al. From pathogen genomes to host plant processes: the power of plant parasitic oomycetes. Genome Biol. 2013;14(6):211. https://doi.org/10.1186/ gb-2013-14-6-211.

Peart JR, Mestre P, Lu R, Malcuit I, Baulcombe DC. NRG1, a CC-NB-LRR protein, together with N, a TIR-NB-LRR protein, mediates resistance against tobacco mosaic virus. Curr Biol. 2005;15(10):968-73. https://doi.org/10. 1016/j.cub.2005.04.053.

Qi T, Seong K, Thomazella DPT, Kim JR, Pham J, Seo E, et al. NRG1 functions downstream of EDS1 to regulate TIR-NLR-mediated plant immunity in Nicotiana benthamiana. Proc Natl Acad Sci U S A. 2018;115(46):E1097987. https://doi.org/10.1073/pnas.1814856115.

Ramirez-Garces D, Camborde L, Pel MJ, Jauneau A, Martinez Y, Neant I, et al. CRN13 candidate effectors from plant and animal eukaryotic pathogens are DNA-binding proteins which trigger host DNA damage response. New Phytol. 2016;210(2):602-17. https://doi.org/10.1111/nph.13774.

Rehmany AP, Gordon A, Rose LE, Allen RL, Armstrong MR, Whisson SC, et al. Differential recognition of highly divergent downy mildew avirulence gene alleles by RPP1 resistance genes from two Arabidopsis lines. Plant Cell. 2005;17(6):1839-50. https://doi.org/10.1105/tpc.105.031807.

Riisberg I, Orr RJS, Kluge R, Shalchian-Tabrizi K, Bowers HA, Patil V, et al. Seven gene phylogeny of heterokonts. Protist. 2009;160(2):191-204. https://doi. org/10.1016/.jprotis.2008.11.004

Saile SC, Jacob P, Castel B, Jubic LM, Salas-Gonzales I, Backer M, et al. Two unequally redundant "helper" immune receptor families mediate Arabidopsis thaliana intracellular "sensor" immune receptor functions. PLoS Biol. 2020;18(9): e3000783. https://doi.org/10.1371/journal.pbio.3000783.

Schornack S, van Damme M, Bozkurt TO, Cano LM, Smoker M, Thines M, et al. Ancient class of translocated oomycete effectors targets the host nucleus. Proc Natl Acad Sci USA. 2010;107(40):17421-6. https://doi.org/ 10.1073/pnas.1008491107.

Shen DY, Tang ZY, Wang C, Wang J, Dong YM, Chen Y, et al. Infection mechanisms and putative effector repertoire of the mosquito pathogenic oomycete Pythium quiyangense uncovered by genomic analysis. PLoS Genet. 2019;15(4): e1008116. https://doi.org/10.1371/journal.pgen.10081 16.

Shen QH, Saijo Y, Mauch S, Biskup C, Bieri S, Keller B, et al. Nuclear activity of MLA immune receptors links isolate-specific and basal disease-resistance responses. Science. 2007;315(5815):1098-103. https://doi.org/10.1126/ science.1136372.

Shirasu K. The HSP90-SGT1 chaperone complex for NLR immune sensors. Annu Rev Plant Biol. 2009;60:139-64. https://doi.org/10.1146/annurev. arplant.59.032607.092906

Stam R, Jupe J, Howden AJM, Morris JA, Boevink PC, Hedley PE, et al. Identification and characterisation CRN effectors in Phytophthora capsici shows modularity and functional diversity. PLoS ONE. 2013;8(3): e59517. https:// doi.org/10.1371/journal.pone.0059517.

Takahashi A, Casais C, Ichimura K, Shirasu K. HSP90 interacts with RAR1 and SGT1 and is essential for RPS2-mediated disease resistance in Arabidopsis. Proc Natl Acad Sci USA. 2003;100(20):11777. https://doi.org/10.1073/ pnas.2033934100.
Thordal-Christensen $\mathrm{H}$. A holistic view on plant effector-triggered immunity presented as an iceberg model. Cell Mol Life Sci. 2020;77(20):3963-76. https://doi.org/10.1007/s00018-020-03515-w.

Tsuda K, Katagiri F. Comparing signaling mechanisms engaged in patterntriggered and effector-triggered immunity. Curr Opin Plant Biol. 2010;13(4):459-65. https://doi.org/10.1016/j.pbi.2010.04.006.

van der Vossen E, Sikkema A, Hekkert BTL, Gros J, Stevens P, Muskens M, et al. An ancient $R$ gene from the wild potato species Solanum bulbocastanum confers broad-spectrum resistance to Phytophthora infestans in cultivated potato and tomato. Plant J. 2003;36(6):867-82. https://doi.org/10.1046/j. 1365-313X.2003.01934.X.

Wang QQ, Han CZ, Ferreira AO, Yu XL, Ye WW, Tripathy S, et al. Transcriptional programming and functional interactions within the Phytophthora sojae RXLR effector repertoire. Plant Cell. 2011;23(6):2064-86. https://doi.org/ 10.1105/tpc.111.086082.

Wu CH, Abd-El-Haliem A, Bozkurt TO, Belhaj K, Terauchi R, Vossen JH, et al. NLR network mediates immunity to diverse plant pathogens. Proc Natl Acad Sci USA. 2017;114(30):8113. https://doi.org/10.1073/pnas.1702041114.

Wu CH, Belhaj K, Bozkurt TO, Birk MS, Kamoun S. Helper NLR proteins NRC2a/b and NRC3 but not NRC1 are required for Pto-mediated cell death and resistance in Nicotiana benthamiana. New Phytol. 2016;209(4):1344-52. https://doi.org/10.1111/nph.13764.

Wu Z, Li M, Dong OX, Xia S, Liang W, Bao Y, et al. Differential regulation of TNL-mediated immune signaling by redundant helper CNLs. New Phytol. 2019;222(2):938-53. https://doi.org/10.1111/nph.15665.

Xu F, Cheng YT, Kapos P, Huang Y, Li X. P-Loop-dependent NLR SNC1 can oligomerize and activate immunity in the nucleus. Mol Plant. 2014;7(12):1801-4. https://doi.org/10.1093/mp/ssu097.

Yin Z, Wang N, Pi L, Li L, Duan W, Wang X, et al. Nicotiana benthamiana LRR-RLP $\mathrm{NbEIX2}$ mediates the perception of an EIX-like protein from Verticillium dahliae. J Integr Plant Biol. 2021;63(5):949-60. https://doi.org/10.1111/ jipb.13031.

Yu G, Xian L, Sang Y, Macho AP. Cautionary notes on the use of Agrobacteriummediated transient gene expression upon SGT1 silencing in Nicotiana benthamiana. New Phytol. 2019;222(1):14-7. https://doi.org/10.1111/nph. 15601.

Zipfel C. Pattern-recognition receptors in plant innate immunity. Curr Opin Immunol. 2008;20(1):10-6. https://doi.org/10.1016/j.coi.2007.11.003.

\section{Publisher's Note}

Springer Nature remains neutral with regard to jurisdictional claims in published maps and institutional affiliations.

Ready to submit your research? Choose BMC and benefit from

- fast, convenient online submission

- thorough peer review by experienced researchers in your field

- rapid publication on acceptance

- support for research data, including large and complex data types

- gold Open Access which fosters wider collaboration and increased citations

- maximum visibility for your research: over $100 \mathrm{M}$ website views per year

At BMC, research is always in progress.

Learn more biomedcentral.com/submissions 